The Egyptian Journal of Hospital Medicine (October 2020) Vol. 81 (6), Page 2121-2128

\title{
Role of Nailfold Capillaroscopy as A Method of Detection of Atherosclerosis in Systemic Lupus Erythematosus Patients
}

Hanan M Farouk ${ }^{1,}$ Fatma M Aboud ${ }^{1}$, Huda T Hussein ${ }^{2}$, Rasha M Mohamed ${ }^{1}$. 1-Department of Internal Medicine and Rheumatology, Faculty of Medicine, Ain Shams University,2-National research center, Cairo, Egypt.

Corresponding Author: Fatma Mohammed Aboud, Mobile: 01286451050, E mail: drfatmaaboud@ gmail.com

\begin{abstract}
Background: Systemic lupus erythematosus (SLE) is a chronic autoimmune disease with increased risk of atherosclerosis. Despite the relative youth of lupus patients, they have risk for the development of premature cardiovascular atherosclerotic diseases. Objective: This work aimed to study the detection of atherosclerosis and the cardiovascular risk in SLE patients by nail fold capillaroscopy and the intima media thickness of the carotid artery.

Patients and methods: This was a cross sectional study included 60 SLE patients. Patients were collected from the Outpatient Clinic and Inpatient of Internal Medicine and Rheumatology Departments within 6 months.

Results: 22 patients (36.7\%) had atherosclerosis, with higher SLE disease activity index (SLEDAI) score (p 0.002). 45.5 $\%$ of them had severe disease activity and $54.5 \%$ had moderate disease activity. Atherosclerotic patients had statistically significant lower capillary density (P 0.001), higher capillary length $(\mathrm{P} 0.008)$, capillary width $(\mathrm{P}<0.001)$ and arterial limb diameter $(\mathrm{P}<0.001)$ with higher prevalence of tortuous $(\mathrm{P}=0.022)$, meandering $(\mathrm{p}=0.014)$ and disorganized capillaries $(\mathrm{P}=$ 0.008). There was statistically significant positive correlation between Intima media thickness (IMT) and disease duration, SLEDAI score, capillary width and arterial limb diameter, while there was statistically significant negative correlation with capillary density. The Arterial limb diameter had highest diagnostic performance in diagnosing atherosclerosis.

Conclusion: Atherosclerosis is common in SLE \& is associated with higher diseases activity. By capillaroscopy, lower density, longer, wider and disorganized capillaries and tortuous \& meandering capillaries were associated with the presence of atherosclerosis.
\end{abstract}

Key words: Capillaroscopy, atherosclerosis, SLE.

\section{INTRODUCTION}

Systemic lupus erythematosus (SLE) is a chronic autoimmune disease usually affects women at reproductive age ${ }^{1}$. Atherosclerosis is an inflammatory process, which affects intima of arteries ${ }^{2}$. Inflammation is involved in the pathogenesis of both lupus and atherogenesis. Endothelial injury caused by several factors including autoantibody production, impaired immune complex clearance and complement activation, which are associated with the pathogenesis of both SLE and coronary artery disease ${ }^{3}$. Quantification of the carotid artery intima -media thickness using ultrasonography has been used to diagnose atherosclerosis ${ }^{4}$. Nail fold capillaroscopy (NFC) is a simple technique, which is useful to detect atherosclerosis and microcirculation 5 . Different abnormal NFC changes are quite common among patients with SLE, and nail fold capillaroscopy is an effective method to monitor such changes. Treatment strategies may change in the different patterns of nail fold capillaroscopy ${ }^{6}$.

NFC can be beneficial in the disease diagnostic process, and the extent of microvascular involvement in SLE may be reflected by the abnormal changes in nail fold capillaroscopy. Severe or moderate pathological changes under NFC were found in all reported SLE patients with internal organ manifestations ${ }^{7}$. This work aimed to study the detection of atherosclerosis and the cardiovascular risk in SLE patients by nail fold capillaroscopy and the intima media thickness of the carotid artery.

\section{PATIENTS AND METHODS}

This was a cross sectional study included 60 SLE patients. Patients were collected from the Outpatient Clinic and Inpatient of Internal Medicine and Rheumatology departments within 6 months.

Inclusion criteria: SLE patients fulfilling the American College of Rheumatology and European League against Rheumatism (ACR / EULAR) 2012 classification criteria for SLE ${ }^{8}$.

Exclusion criteria: Patients with diabetes mellitus, hypertension, obese patients "BMI more than 30" and patients with other connective tissue diseases were excluded from the study.

Ethical consideration: The study was approved by Local Research Ethical Committee of Ain Shams University and conforms to the provisions of the Declaration of Helsinki in 1995. Informed consent from all patients was obtained after explaining the aim of the work and study design.

Demographic data including age, sex and disease duration, clinical manifestations including musculoskeletal, constitutional, renal, neurological, hematological, mucocutanous, cardiovascular, 
pulmonary, and gastrointestinal and drugs used for treatment including steroids, hydroxychloroquine and immunosuppressive drugs were carefully recorded. Disease activity was assessed using SLEDAI score ${ }^{9}$. Activity categories were defined as follow: Score 0 considered non active, score 1-5 considered as mild activity, score 6-10 considered as moderate activity and score 11-19 considered as high activity and score 20 or more considered asd very high activity ${ }^{9}$.

Laboratory investigations: including complete blood picture $(\mathrm{CBC})$, erythrocyte sedimentation rate (ESR), C-reactive protein (CRP), kidney function test, liver enzymes, lipid profile, antinuclear antibody (ANA), anti-double stranded DNA antibody (anti ds-DNA), antiphospholipid antibodies, complement 3 and complement 4.

Radiological investigations: Including nail fold capillary microscopy (NFC) and carotid duplex were done to all included patients.

NFC was carried out using a videocapillaroscopy with a probe (magnification $\times 200$ ). Examination was done for the eight fingers thumb was excluded. All the images were registered and collected using a dedicated software system (Blu Vision). NFC was performed according to the standard method using high magnification videaocapillaroscopy, at room temperature using light olive oil as lubricants ${ }^{10}$.

The following parameters were considered, comment on the shape of the capillaries (normal hairpin or abnormal shape eg. meandering, corkscrew or tortuous capillaries), the diameter of the capillaries is determined (dilated loops considered if loop diameter is more than 20 $\mu \mathrm{m}$ and mega capillaries if $\geq 50 \mu \mathrm{m}$ ) and capillary length (normal or elongated $\geq 300 \mu \mathrm{m}$ ). Comment on mean capillary density (low capillary density defined as number of capillaries below 7 per linear $\mathrm{mm}$ ), avascular area (inter capillary distance $\geq 500 \mu \mathrm{m}$ ) and comment on the presence or absence of microhemorrhage was done ${ }^{10}$. Patients were also subjected for carotid artery duplex to detect the presence or absence of increased carotid intima -media thickness (IMT) through measuring IMT by B-mode ultrasound using Toshiba Xario (USA) using 7.5 MHs probe. For each segment, the maximal value of IMT is selected and the final IMT considered is the average of IMT values at the 12 examined sites. Patients with IMT < $1 \mathrm{~mm}$ were considered non-atherosclerotic, while those with IMT $>1 \mathrm{~mm}$ were considered atherosclerotic ${ }^{4}$.

\section{Statistical analysis}

The Statistical package for Social Science (SPSS version 20) was used. Data were presented and suitable analysis was performed. Unpaired student T-test was used to compare between two independent groups regarding quantitative data. Chi-square was used to compare qualitative variables. $\mathrm{P}$ value $\leq 0.05$ was considered significant. Pearson's correlation coefficient (r) test was used for correlating data.

\section{RESULTS}

Among the studied 60 patients $58(96.7 \%)$ were females and $2(3.3 \%)$ were males. They had a mean age of $42.8 \pm 2.2$ years and a mean disease duration of $6.0 \pm 2.2$ years (Table 1).

Table (1): Descriptive data of the studied patients

\begin{tabular}{|c|c|c|c|}
\hline \multicolumn{3}{|c|}{ Characteristics } & Mean \pm SD \\
\hline \multicolumn{3}{|c|}{ Age/ years } & $42.8 \pm 5.4$ \\
\hline \multicolumn{3}{|c|}{ Disease duration/ years } & $6.0 \pm 3.3$ \\
\hline \multirow{3}{*}{ sex } & & $\mathrm{N}$ \\
\hline & \multicolumn{2}{|l|}{ male } & 2 \\
\hline & \multicolumn{2}{|c|}{ female } & 58 \\
\hline \multirow{2}{*}{\multicolumn{3}{|c|}{ SLEDAI score }} & $17.6 \pm 1.4$ \\
\hline & & & $\mathrm{N}$ \\
\hline \multirow{3}{*}{ Severity } & \multicolumn{2}{|c|}{ Mild } & 20 \\
\hline & \multicolumn{2}{|c|}{ Moderate } & 26 \\
\hline & \multicolumn{2}{|c|}{ Severe } & 14 \\
\hline \multicolumn{3}{|c|}{ Triglycerides (mg/dL) } & $140.3 \pm 14.8$ \\
\hline \multicolumn{3}{|c|}{ LDL (mg/dL) } & $131.5 \pm 14.2$ \\
\hline \multicolumn{3}{|c|}{ HDL (mg/dL) } & $59.2 \pm 10.6$ \\
\hline \multicolumn{3}{|c|}{ ESR (mg/dL) } & $52.3 \pm 9.3$ \\
\hline \multicolumn{3}{|c|}{ CRP (mg/dL) } & $23.2 \pm 7.0$ \\
\hline \multicolumn{3}{|c|}{$\mathrm{C3}$ (mg/dL) } & $58.8 \pm 10.6$ \\
\hline \multicolumn{3}{|c|}{$\mathrm{C4}$ (mg/dL) } & $14.1 \pm 3.0$ \\
\hline & & & $\mathrm{N}$ \\
\hline \multirow{2}{*}{\multicolumn{2}{|c|}{ Anti dsDNA }} & Positive & 42 \\
\hline & & Negative & 18 \\
\hline \multirow{2}{*}{\multicolumn{2}{|c|}{ Antiphospholipid }} & Positive & 0 \\
\hline & & \begin{tabular}{|l|} 
Negative \\
\end{tabular} & 60 \\
\hline \multicolumn{3}{|c|}{ Capillary Density $(7.3-10.3 \mathrm{~mm})$} & $8.9 \pm 1.0$ \\
\hline \multicolumn{3}{|c|}{ Capillary Length $(92.0-295.0 \mu \mathrm{m})$} & $141.5 \pm 12.7$ \\
\hline \multicolumn{3}{|c|}{ Width diameter $(27.0-59.5 \mu \mathrm{m})$} & $26.5 \pm 4.3$ \\
\hline \multirow{2}{*}{\multicolumn{3}{|c|}{$\begin{array}{l}\text { Arterial loop diameter } \\
(\mathbf{7 . 0}-17.0 \mu \mathrm{m})\end{array}$}} & $14.8 \pm 3.9$ \\
\hline & & & $\mathbf{N}$ \\
\hline \multirow{5}{*}{\multicolumn{2}{|c|}{ Shape }} & Meandering & 32 \\
\hline & & Tortuous & 28 \\
\hline & & Corkscrew & 10 \\
\hline & & Bushy & 4 \\
\hline & & Hairpin & 4 \\
\hline \multicolumn{3}{|c|}{ Hemorrhage } & 8 \\
\hline \multicolumn{3}{|c|}{ Subpaplliary Plexus } & 28 \\
\hline Avascula & treas & & 0 \\
\hline Distribl & & sorganization & 36 \\
\hline Distrio & & Organization & 24 \\
\hline
\end{tabular}

According to the intima media thickness assessed

by carotid duplex, patients were classified into 
atherosclerotic group [22 patients $(36.7 \%)]$ and non-

atherosclerotic group [38 (63.3\%)].

Table (2): Comparison between both groups regarding demographic data

\begin{tabular}{|c|c|c|c|c|c|c|}
\hline \multicolumn{2}{|l|}{ Variables } & \multirow{2}{*}{$\begin{array}{c}\begin{array}{c}\text { Atherosclerotic } \\
(\mathbf{N}=\mathbf{2 2})\end{array} \\
46.0 \pm 5.3 \\
\end{array}$} & \multirow{2}{*}{$\begin{array}{c}\begin{array}{c}\text { Non- } \\
\text { atherosclerotic } \\
(\mathbf{N}=\mathbf{3 8})\end{array} \\
41.0 \pm 4.7\end{array}$} & \multirow{2}{*}{$\begin{array}{c}\begin{array}{c}\text { Test } \\
\text { value }\end{array} \\
t=\end{array}$} & \multirow{3}{*}{$\begin{array}{c}\text { P-value } \\
0.010^{*}\end{array}$} & \multirow{3}{*}{$\begin{array}{r}\text { Sig. } \\
S\end{array}$} \\
\hline \multirow{2}{*}{ Age (years) } & Mean \pm SD & & & & & \\
\hline & Range & $39.0-57.0$ & $34.0-49.0$ & 3.098 & & \\
\hline \multirow{2}{*}{$\begin{array}{l}\text { Age of disease } \\
\text { onset (years) }\end{array}$} & Mean \pm SD & $38.0 \pm 2.0$ & $36.2 \pm 2.1$ & \multirow{2}{*}{$\begin{array}{c}\mathrm{t}= \\
2.696\end{array}$} & \multirow[b]{2}{*}{$0.022 *$} & \multirow{2}{*}{$\mathbf{S}$} \\
\hline & Range & $35.0-42.0$ & $33.0-40.0$ & & & \\
\hline \multirow{2}{*}{$\begin{array}{l}\text { Disease duration } \\
\text { (years) }\end{array}$} & Mean \pm SD & $8.0 \pm 3.4$ & $4.8 \pm 2.7$ & \multirow{2}{*}{$\begin{array}{c}\mathrm{t}= \\
3.218\end{array}$} & \multirow[b]{2}{*}{$0.008 *$} & \multirow{2}{*}{$\mathbf{S}$} \\
\hline & Range & $4.0-15.0$ & $1.0-9.0$ & & & \\
\hline \multirow{2}{*}{ Sex } & Male & $0(0.0 \%)$ & $2(5.3 \%)$ & \multirow{2}{*}{$\begin{array}{c}\chi^{2}= \\
0.689\end{array}$} & \multirow[b]{2}{*}{0.780} & \multirow{2}{*}{ NS } \\
\hline & Female & $22(100.0 \%)$ & $36(94.7 \%)$ & & & \\
\hline
\end{tabular}

On comparing both groups, atherosclerotic patients were statistically significantly older $(\mathrm{p}=0.012)$ with statistically significant longer disease duration $(\mathrm{p}=0.009)$ (Table 2).

Atherosclerotic patients had statistically significant higher SLEDAI score (p 0.002), higher frequency of moderate (P 0.001) and severe (P 0.009) disease activity (Table 3).

Table (3): Comparison between both groups regarding disease activity

\begin{tabular}{|c|c|c|c|c|c|c|}
\hline Variables & & $\begin{array}{l}\text { Atherosclerotic } \\
\qquad(\mathrm{N}=22)\end{array}$ & $\begin{array}{c}\text { Non- } \\
\text { atherosclerotic } \\
(\mathbf{N}=38) \\
\end{array}$ & $\begin{array}{c}\text { Test } \\
\text { value }\end{array}$ & P-value & Sig. \\
\hline \multirow{2}{*}{$\begin{array}{l}\text { SLEDAI } \\
\text { score }\end{array}$} & Mean \pm SD & $11.6 \pm 3.1$ & $7.0 \pm 3.8$ & \multirow{2}{*}{$\begin{array}{c}\mathrm{t}= \\
3.966\end{array}$} & \multirow[t]{2}{*}{$0.002 *$} & \multirow[t]{2}{*}{$\mathbf{S}$} \\
\hline & Range & $7.0-17.0$ & $4.0-15.0$ & & & \\
\hline \multirow[t]{3}{*}{ Severity } & Mild & $0(0.0 \%)$ & $20(52.7 \%)$ & \multirow{3}{*}{$\begin{array}{c}\chi^{2}= \\
11.426\end{array}$} & $0.002 *$ & $\mathbf{S}$ \\
\hline & Moderate & $12(54.5 \%)$ & $14(36.8 \%)$ & & 0.001* & $\mathbf{S}$ \\
\hline & Severe & $10(45.5 \%)$ & $4(10.5 \%)$ & & $0.007 *$ & $\mathbf{S}$ \\
\hline
\end{tabular}

Also, there was statistically significant difference between atherosclerotic and non-atherosclerotic SLE patients as regards the frequency of arthritis ( P 0.007) and nephritis ( P0.007) being higher in the atherosclerotic patients. Moreover, atherosclerotic patients had statistically significant higher doses of steroids $(\mathrm{P}<0.001)$ (Table 4$)$.

Table (4): Comparison between both groups regarding clinical manifestations and drug intake

\begin{tabular}{|l|c|c|c|c|}
\hline Parameters & $\begin{array}{c}\text { Atherosclorosis } \\
(\mathbf{n = 2 2})\end{array}$ & $\begin{array}{c}\text { Non } \\
\text { atherosclerotic } \\
(\mathbf{n = 3 8 )}\end{array}$ & Test & p-value \\
\hline Corticosteroid (mg) & $31.29 \pm 5.32$ & $10.43 \pm 1.77$ & $t=22.251$ & $<0.001^{* *}$ \\
\hline Immunosuppressive & $13(59.1 \%)$ & $17(44.7 \%)$ & $x^{2}=0.651$ & 0.419 \\
\hline Hydroxychloroquine & $5(22.7 \%)$ & $24(63.2 \%)$ & $x^{2}=7.602$ & $0.006^{*}$ \\
\hline Arthritis & $15(68.2 \%)$ & $11(28.9 \%)$ & $x^{2}=7.237$ & $0.007^{*}$ \\
\hline rash & $7(31.8 \%)$ & $11(28.9 \%)$ & $x^{2}=0.003$ & 0.995 \\
\hline Nephritis & $13(59.1 \%)$ & $8(21.1 \%)$ & $x^{2}=7.249$ & $0.007 *$ \\
\hline Serositis & $4(18.2 \%)$ & $7(18.4 \%)$ & $x^{2}=0.107$ & 0.744 \\
\hline CNS & $2(9.1 \%)$ & $3(7.9 \%)$ & $x^{2}=0.104$ & 0.747 \\
\hline
\end{tabular}

Regarding nail fold capillaroscopic findings, atherosclerotic patients showed statistically significant higher prevalence of longer capillaries $(\mathrm{p}=0.009)$, lower capillary density $(\mathrm{p}=0001)$, wider capillary diameter $(\mathrm{p}=0.002)$ and larger arterial limb diameter $(\mathrm{p}=0.001)$. In addition, meandering, tortuous and disorganization were significantly more frequent in atherosclerosis cases (P 0.014), (P 0.022), (P 0.008) respectively (Table 5) 
Table (5): Comparison between both groups regarding laboratory data and nail fold capillaroscopic findings

\begin{tabular}{|c|c|c|c|c|c|c|}
\hline Variables & & $\begin{array}{c}\text { Atherosclerosis } \\
(\mathbf{N}=22)\end{array}$ & $\begin{array}{c}\text { No atherosclerosis } \\
(\mathbf{N}=\mathbf{3 8})\end{array}$ & Test value & P-value & Sig. \\
\hline \multirow{2}{*}{$\begin{array}{l}\text { Cholesterol } \\
\text { (mg/dL) }\end{array}$} & Mean \pm SD & $222.3 \pm 24.1$ & $213.4 \pm 24.7$ & \multirow{2}{*}{$\begin{array}{c}\mathrm{t}= \\
1.097\end{array}$} & \multirow{2}{*}{$\hat{\wedge}$} & \multirow{2}{*}{ NS } \\
\hline & Range & $183.0-257.0$ & $174.0-264.0$ & & & \\
\hline \multirow{2}{*}{$\begin{array}{l}\text { Triglyceride } \\
\text { s (mg/dL) }\end{array}$} & Mean \pm SD & $142.6 \pm 14.5$ & $138.9 \pm 15.2$ & \multirow{2}{*}{$\begin{array}{c}\mathrm{t}= \\
0.749\end{array}$} & \multirow{2}{*}{$\hat{\wedge}$} & \multirow{2}{*}{ NS } \\
\hline & Range & $117.0-163.0$ & $115.0-173.0$ & & & \\
\hline \multirow{2}{*}{$\begin{array}{l}\text { LDL } \\
(\mathrm{mg} / \mathrm{dL})\end{array}$} & Mean \pm SD & $140.4 \pm 13.1$ & $126.8 \pm 12.8$ & \multirow{2}{*}{$\begin{array}{c}t= \\
3.203\end{array}$} & \multirow{2}{*}{$\hat{0}$} & \multirow{2}{*}{$\mathbf{S}$} \\
\hline & Range & $115.0-157.0$ & $104.0-156.0$ & & & \\
\hline \multirow{2}{*}{$\begin{array}{l}\text { HDL } \\
(\mathrm{mg} / \mathrm{dL})\end{array}$} & Mean \pm SD & $57.4 \pm 11.0$ & $60.2 \pm 10.5$ & \multirow{2}{*}{$\begin{array}{c}\mathrm{t}= \\
-0.810\end{array}$} & \multirow{2}{*}{$\stackrel{\wedge}{0.414}$} & \multirow{2}{*}{ NS } \\
\hline & Range & $42.0-78.0$ & $41.0-84.0$ & & & \\
\hline \multirow{3}{*}{$\begin{array}{l}\text { ESR } \\
(\mathrm{mg} / \mathrm{dL}) \\
\text { CRP } \\
(\mathrm{mg} / \mathrm{dL})\end{array}$} & Mean \pm SD & $56.4 \pm 10.2$ & $49.2 \pm 8.4$ & \multirow{2}{*}{$\begin{array}{c}\mathrm{t}= \\
2.884\end{array}$} & \multirow{2}{*}{$\hat{\wedge}$} & \multirow{2}{*}{$\mathbf{S}$} \\
\hline & Range & $42.0-72.0$ & $41.0-76.0$ & & & \\
\hline & Mean \pm SD & $24.8 \pm 6.4$ & $22.3 \pm 5.3$ & $\begin{array}{c}\mathrm{t}= \\
1.175\end{array}$ & $\hat{\wedge}$ & NS \\
\hline \multirow{2}{*}{ C3 (mg/dL) } & Mean \pm SD & $56.6 \pm 10.5$ & $60.1 \pm 10.6$ & \multirow{2}{*}{$\begin{array}{c}t= \\
-0.978\end{array}$} & \multirow{2}{*}{$\begin{array}{c}\wedge \\
0.342\end{array}$} & \multirow{2}{*}{ NS } \\
\hline & Range & $39.0-78.0$ & $39.0-79.0$ & & & \\
\hline \multirow{2}{*}{ C4 (mg/dL) } & Mean \pm SD & $13.6 \pm 2.7$ & $14.4 \pm 3.2$ & \multirow{2}{*}{$\begin{array}{c}t= \\
-0.796\end{array}$} & \multirow{2}{*}{$\hat{\wedge}$} & \multirow{2}{*}{ NS } \\
\hline & Range & $9.0-19.0$ & $8.0-22.0$ & & & \\
\hline AntidsDNA & Positive & $16(72.7 \%)$ & $26(68.4 \%)$ & $\chi^{2}=$ & 0683 & NS \\
\hline Antı dsDNA & Negative & $6(27.3 \%)$ & $12(31.6 \%)$ & 0.689 & 0.683 & NS \\
\hline Capillary & Mean \pm SD & $8.3 \pm 0.5$ & $9.3 \pm 1.1$ & $t=$ & & \\
\hline $\begin{array}{l}\text { Density } \\
(\mathbf{m m})\end{array}$ & Range & $8.0-9.0$ & $8.0-12.0$ & 4.278 & $0.001 *$ & $\mathbf{S}$ \\
\hline Capillary & Mean \pm SD & $149.2 \pm 15.3$ & $137.0 \pm 8.6$ & $t=$ & & \\
\hline $\begin{array}{l}\text { Length } \\
(\mu \mathrm{m})\end{array}$ & Range & $111.0-173.0$ & $124.0-157.0$ & 3.230 & 0.008* & $\mathbf{S}$ \\
\hline Width & Mean \pm SD & $31.0 \pm 5.9$ & $22.8 \pm 4.2$ & $t=$ & $<$ & \\
\hline $\begin{array}{l}\text { diameter } \\
(\mu \mathrm{m})\end{array}$ & Range & $19.0-37.0$ & $15.0-28.0$ & 6.582 & $0.001 *$ & $\mathbf{S}$ \\
\hline Arterial loop & Mean \pm SD & $18.7 \pm 2.2$ & $12.6 \pm 2.7$ & & $<$ & \\
\hline & Range & $14.0-22.0(\mu \mathrm{m})$ & $9.0-16.0(\mu \mathrm{m})$ & $t=7.304$ & $0.001 *$ & $\mathbf{S}$ \\
\hline & Meandering & $18(81.8 \%)$ & $14(36.8 \%)$ & $\begin{array}{c}\chi^{2}= \\
6.511\end{array}$ & $0.014 *$ & $\mathbf{S}$ \\
\hline & Tortuous & $16(72.7 \%)$ & $12(31.6 \%)$ & $\chi^{2}=5.450$ & 0.022* & $\mathbf{S}$ \\
\hline Shapes & Corkscrew & $6(27.3 \%)$ & $4(10.5 \%)$ & $\begin{array}{c}\chi^{2}= \\
1.618\end{array}$ & 0.201 & NS \\
\hline & Bushy & $2(9.1 \%)$ & $2(5.3 \%)$ & $\chi^{2}=0.189$ & 0.582 & NS \\
\hline & Hairpin & $0(0.0 \%)$ & $4(10.5 \%)$ & $\chi^{2}=1.427$ & 0.225 & NS \\
\hline Haemorrhage & & $6(27.3 \%)$ & $2(5.3 \%)$ & $\chi^{2}=3.359$ & 0.074 & NS \\
\hline Sub papillary & plexus & $10(45.4 \%)$ & $18(47.3 \%)$ & $\chi^{2}=3.359$ & 0.074 & NS \\
\hline Dictrik & Disorganization & $20(90.9 \%)$ & $16(42.1 \%)$ & $\chi^{2}=$ & $0008 *$ & $\mathbf{S}$ \\
\hline Distridou & Organization & $4(9.1 \%)$ & $22(57.9 \%)$ & 7.951 & & $\mathbf{S}$ \\
\hline
\end{tabular}

Correlating carotid IMT with capillaroscopic findings, disease activity and various laboratory data documented statistically significant positive correlation between IMT and age ( $r=0.427, \mathrm{P} 0.037)$, disease duration $(\mathrm{r}=0.484, \mathrm{P} 0.017)$, SLEDAI score ( $r=0.536, \mathrm{P} 0.008)$, capillary width $(\mathrm{r}=0.692, \mathrm{P}<0.001)$ and arterial limb diameter $(\mathrm{r}=0.846, \mathrm{P}<0.001)$. While, there was statistically significant negative correlation with capillary density $(\mathrm{r}=-0.728, \mathrm{P}<0.001)($ Fig $1,2,3)$. 


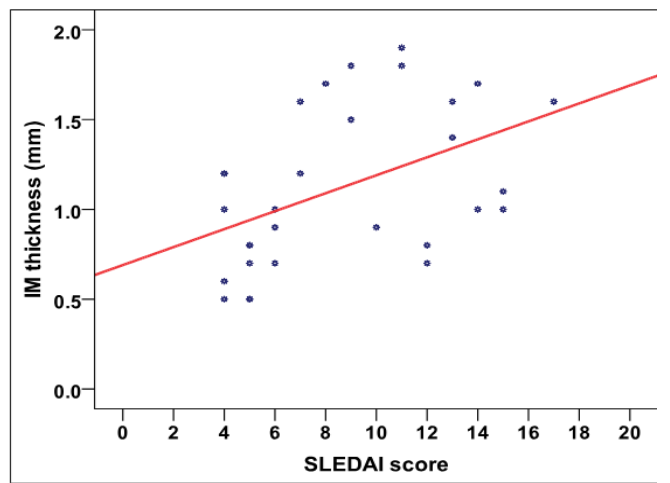

Figure (1): Correlation between IM thickness and SLEDAI score

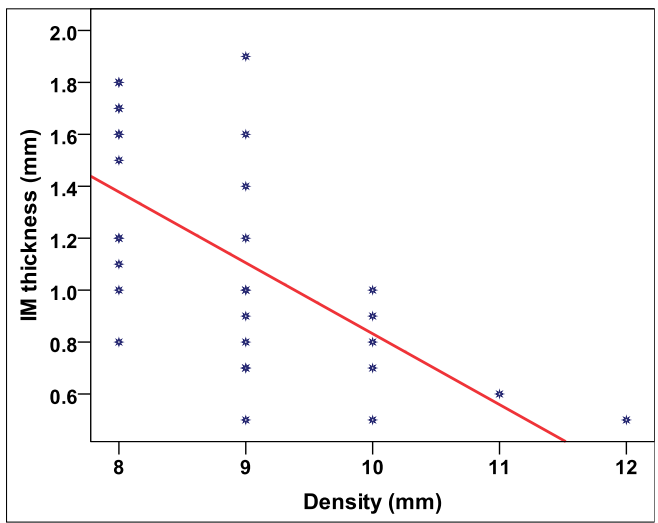

Figure (2): Correlation between IMT and capillary density

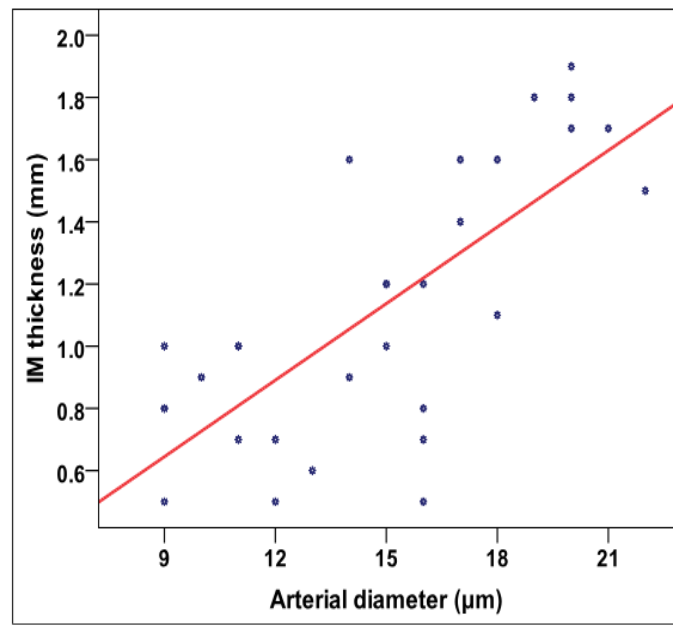

Figure (3): correlation between IMT and arterial limb diameter

In addition, the arterial limb diameter found to has the highest diagnostic performance in diagnosing atherosclerosis. The arterial diameter $\geq 17.0 \mu \mathrm{m}$ had highest diagnostic characteristics in diagnosing atherosclerosis (Tables 6, 7).

Table (6): Diagnostic performance of nail fold capillaroscopy in diagnosing atherosclerosis

\begin{tabular}{|l|c|c|c|c|c|}
\hline \multicolumn{1}{|c|}{ Factors } & AUC & SE & P-value & 95\% CI & Cut off \\
\hline Density & 0.840 & 0.078 & $\mathbf{0 . 0 0 2} *$ & $0.840-0.078$ & $\geq 9.0$ \\
\hline Length & 0.833 & 0.093 & $\mathbf{0 . 0 0 3} *$ & $0.833-0.093$ & $\geq 144.0$ \\
\hline Width & 0.825 & 0.075 & $\mathbf{0 . 0 0 3} *$ & $0.825-0.075$ & $\geq 55.0$ \\
\hline Arterial diameter & 0.964 & 0.037 & $<\mathbf{0 . 0 0 1} *$ & $0.964-0.037$ & $\geq 17.0$ \\
\hline
\end{tabular}

AUC: Area under curve, SE: Standard error, CI: Confidence interval, *significant 
Table (7): Diagnostic characteristics of nail fold capillaroscopy in diagnosing atherosclerosis

\begin{tabular}{|l|c|c|c|c|c|c|}
\hline \multicolumn{1}{|c|}{ Characters } & $\begin{array}{c}\text { Density } \\
\mathbf{2 9 . 0}\end{array}$ & $\begin{array}{c}\text { Length } \\
\mathbf{1 4 4 . 0}\end{array}$ & $\begin{array}{c}\text { Width } \\
\mathbf{5 5 5 . 0}\end{array}$ & $\begin{array}{c}\text { Diameter } \\
\mathbf{1 1 7 . 0}\end{array}$ & Meandering & Tortous \\
\hline Sensitivity & $100.0 \%$ & $81.8 \%$ & $54.5 \%$ & $90.9 \%$ & $81.8 \%$ & $72.7 \%$ \\
\hline Specificity & $5.3 \%$ & $84.2 \%$ & $89.5 \%$ & $100.0 \%$ & $63.2 \%$ & $68.4 \%$ \\
\hline DA & $40.0 \%$ & $83.3 \%$ & $76.7 \%$ & $96.7 \%$ & $70.0 \%$ & $70.0 \%$ \\
\hline YI & $5.3 \%$ & $66.0 \%$ & $44.0 \%$ & $\mathbf{9 0 . 9 \%}$ & $45.0 \%$ & $41.1 \%$ \\
\hline PPV & $37.9 \%$ & $75.0 \%$ & $75.0 \%$ & $100.0 \%$ & $56.3 \%$ & $57.1 \%$ \\
\hline NPV & $100.0 \%$ & $88.9 \%$ & $77.3 \%$ & $95.0 \%$ & $85.7 \%$ & $81.3 \%$ \\
\hline LR+ & 1.06 & 5.18 & 5.18 & $>100.0$ & 2.22 & 2.30 \\
\hline LR- & 0.00 & 0.22 & 0.51 & 0.09 & 0.29 & 0.40 \\
\hline LR & $>100.0$ & 24.00 & 10.20 & $>100.0$ & 7.71 & 5.78 \\
\hline & Corkscrew & $\mathbf{B u s h y}$ & Enlargment & Hemorrhage & Plexus & Disorganization \\
\hline Sensitivity & $27.3 \%$ & $9.1 \%$ & $36.4 \%$ & $27.3 \%$ & $9.1 \%$ & $90.9 \%$ \\
\hline Specificity & $89.5 \%$ & $94.7 \%$ & $84.2 \%$ & $94.7 \%$ & $94.7 \%$ & $57.9 \%$ \\
\hline DA & $66.7 \%$ & $63.3 \%$ & $66.7 \%$ & $70.0 \%$ & $63.3 \%$ & $70.0 \%$ \\
\hline YI & $16.7 \%$ & $3.8 \%$ & $20.6 \%$ & $22.0 \%$ & $3.8 \%$ & $48.8 \%$ \\
\hline PPV & $60.0 \%$ & $50.0 \%$ & $57.1 \%$ & $75.0 \%$ & $50.0 \%$ & $55.6 \%$ \\
\hline NPV & $68.0 \%$ & $64.3 \%$ & $69.6 \%$ & $69.2 \%$ & $64.3 \%$ & $91.7 \%$ \\
\hline LR+ & 2.59 & 1.73 & 2.30 & 5.18 & 1.73 & 2.16 \\
\hline LR- & 0.81 & 0.96 & 0.76 & 0.77 & 0.96 & 0.16 \\
\hline LR & 3.19 & 1.80 & 3.05 & 6.75 & 1.80 & 13.75 \\
\hline
\end{tabular}

YI: Youden's index, DA: Diagnostic accuracy, PPV: Positive Predictive value, NPV: Negative Predictive value, LR+: Positive likelihood ratio, LR-: Negative likelihood ratio, LR: Diagnostic odd ratio.

\section{DISCUSSION}

SLE is an autoimmune disease characterized by exacerbations and remissions. Autoantibody production and immune complex deposition leads to immunological self- tolerance breakdown, release inflammatory cytokines and activation of T cells ${ }^{11}$. The inflammatory mediators may contribute to the formation of atherosclerosis in SLE, which makes SLE a risk factor to atherosclerosis ${ }^{12}$.

Nail fold capillaroscopy (NFC) is a safe, noninvasive technique used to detect peripheral microangiopathy. It is also defined to have diagnostic and prognostic value. Microvascular involvement is an important feature in SLE. In patients with SLE, micro vascular changes may be evaluated by NFC ${ }^{13}$. NFC may be used in personalized medicine as an adjunct in assessing SLE activity, which may help prognosticate risk of serious complications ${ }^{14}$.

This study included 60 patients; 58 patients were females and 2 males with a mean age of $42.8 \pm 5.4$. These results run in agreement with Parks et al. ${ }^{15}$ who reported that SLE is more common in females than males.

Accelerated atherosclerosis is often considered a general feature of SLE and is, in similarity to the general population assumed to be the main cause of premature cardiovascular disease (CVD) ${ }^{16}$. In this study, 22 $(36.7 \%)$ patients had atherosclerosis identified by increased intima media thickness. This comes in agreement with khairy et al. ${ }^{17}$ and McMahon et al. ${ }^{18}$ as it concluded that SLE patients had significantly increased IMT compared to general population. In addition, Gustafsson et al. ${ }^{16}$ concluded that atherosclerosis is common among SLE patients with a prevalence of 16$20 \%$. Furthermore, in a study conducted by Katz et al. ${ }^{19}$ comparing hospitalized lupus patients with age and sex matched non-SLE patients, they concluded that SLE was associated with more prevalence of atherosclerotic cardiovascular complications. Age play a very important risk factor for atherosclerosis ${ }^{19}$, in a study conducted by Doria $\boldsymbol{e t}$ al. ${ }^{20}$, they reported that SLE patients with increased IMT were significantly older. This is in harmony with results of the current study as we found that atherosclerotic SLE patients were older in age.

As regards laboratory investigations in the present study, higher ESR was detected in atherosclerotic group in comparison to non-atherosclerotic. However, there was no difference regarding the rest of the $\mathrm{C} 3, \mathrm{C} 4$ and ds-DNA. This is consistent with Gustafsson et al. ${ }^{16}$ who found no differences between lupus patients with carotid plaque and those without regarding ds DNA seropositivity; however, they detected higher level of C4 in patients with carotid plaque.

Although serum cholesterol and triglycerides were higher in the atherosclerotic than nonatherosclerotic patients but it did not reach the statistical significance. This disagrees with khairy et $\boldsymbol{a l} .{ }^{17}$ who 
stated that SLE patients with increased IMT had dyslipidemia with raised TG, LDL and low HDL level. That disagreement may be due to small sample size of our study.

SLE disease activity has been shown to be an independent predictor of organ damage and mortality, it is also considered as a lupus -specific risk factor for premature atherosclerosis ${ }^{18}$.

In the current study, the statistically significant higher frequency of arthritis and nephritis with higher doses of steroids in the atherosclerotic SLE patients compared to the non-atherosclerotic may reflect more sever disease and organ damage. In addition, the statistically significant higher SLEDAI score in atherosclerotic patients and higher frequency of moderate and severe disease activity with presence of statistically significant positive correlation between the carotid IMT and SLEDAI score. All these previous results support that higher disease activity is strongly associated with presence of atherosclerosis in SLE. That association was concluded also by khairy et $\boldsymbol{a l}^{17}$ who stated that SLEDAI score was higher in patients with atherosclerosis. These results come in agreement with Belibou et al. ${ }^{21}$ study who concluded that there was a positive correlation between IMT and SLEDAI score. However, Manzi et al. $^{22}$ found an inverse relationship between disease activity and carotid plaque. In addition, our results go hand in hand with the results of Soliman $e t$ $a^{23}$ who concluded that $50 \%$ of patients with high SLEDAI score have increased IMT, while $25 \%$ of the patients who had moderate increase in SLEDAI score had increased IMT and the results were highly significant.

Based on the comparison between the two groups regarding nail fold capillary microscopic results, results of this study showed that the capillary length, width and arterial diameter were statistically significantly higher in atherosclerotic patients, while capillary density was statistically significantly lower in those patients. In addition, meandering, tortuous and disorganized capillaries were significantly more frequent among atherosclerotic cases. However, there was no statistically significant difference among the studied groups regarding the presence of corkscrew or bushy capillaries. On the other hand, results of Ragab et al. ${ }^{24}$ reported that meandering, tortuous, corkscrew, bushy capillaries were found in atherosclerotic SLE patient by $45 \%, 16 \%, 15 \%$ and $7.5 \%$, while capillary enlargement and hemorrhage were found to be $22.5 \%$ and $10 \%$. In addition, results of this study are supported by Yuksel et $\boldsymbol{a l} .{ }^{25}$ studied 25 SLE patients and demonstrated that the capillaroscopic abnormalities included dilatation \& hemorrhage and tortuosity that were 5.7 times more frequent among those patients with atherosclerosis.

Results of current study showed that the arterial limb diameter had highest diagnostic performance in diagnosing atherosclerosis with arterial diameter $\geq 17.0$ um. This is nearly similar to Ragab et al. ${ }^{24}$ who reported that meandering of capillaries and arterial limb diameter are of most important values to diagnose atherosclerosis in SLE.

On correlating IMT with various parameter of nail fold capillary microscopy, there was significant positive correlation with capillary length, capillary width and arterial limb diameter and inverse correlation with capillary density. However, we didn't find studies discussing this correlation yet.

\section{CONCLUSIONS}

We concluded that atherosclerosis is common in SLE and it is associated with higher disease activity. In addition, nail fold capillary microscopy is helpful in the detection of atherosclerosis in SLE patients, the capillaroscopic findings may reflect the changes of the intima media thickness, and it may be correlated with these changes.

Limitations of the study: Small sample size is the main limitation of this study.

\section{REFERENCES}

1. Hedrich C, Smith E, Beresford M (2017): Juvenile-onset systemic lupus erythematosus (SLE)-Pathophysiological concepts and treatment options. Best Pract Res Clin Rheumatol., 31 (4): 488-504.

2. Vaccarezza M, Balla C, Rizzo P (2018): Atherosclerosis as an inflammatory disease: Doubts? No more. Int J Cardiol Heart Vasc., 19: 1-2.

3. Rhew E, Ramsey-Goldman $R$ (2006): Premature atherosclerotic disease in systemic lupus erythematosus role of inflammatory mechanisms. Autoimmune Rev., 5 (2): 101-105.

4. Stein J, Korcarz C, Hurst R, Lonn E, Kendall C, Mohler E et al. (2008): Use of carotid ultrasound to identify subclinical vascular disease and evaluate cardiovascular disease risk: a consensus statement from the American Society of Echocardiography Carotid Intima-Media Thickness Task Force. Endorsed by the Society for Vascular Medicine. J Am Soc Echocardiogr., 21 (2): 93111.

5. Smith V, Herrick A, Ingegnoli $F$, Damjanov $N$, De Angelis $\mathrm{R}$ et al. (2020): Standardisation of nailfold capillaroscopy for the assessment of patients with Raynaud's phenomenon and systemic sclerosis. Autoimmunity Reviews, 19 (3): 102458. 
6-Raeeskarami S, Namazi N, Assari R, Najafizadeh S, Hassannejad Z, Ziaee V et al. (2020): The Comparison of Nailfold Capillaroscopy between Juvenile Systemic Lupus Erythematosus and Healthy Controls: Correlation with Laboratory and Clinical

Parameters. https://doi.org/10.1155/2020/7631958.

7.Cutolo M, Melsens K, Wijnant S, Ingegnoli F, Thevissen K,De Keyser F et al. (2018): Nailfold capillaroscopy in systemic lupus erythematosus: A systematic review and critical appraisal. Autoimmun Rev., 17 (4): 344-352.

8. Petri M, Orbai A, Alarcón G, Gordon C, Merrill J, Fortin $P$ et al. (2012): Derivation and Validation of Systemic Lupus International Collaborating Clinics Classification Criteria for Systemic Lupus Erythematosus. Arthritis Rheum., 64 (8): 2677-2686.

9. Bombardier C, Gladman D, Urowitz M , Caron D, Chang C (1992): Derivation of the SLEDAI. A disease activity index for lupus patients. The Committee on Prognosis Studies in SLE, Arthritis and Rheumatism, 35 (6): 630-640.

10. Parker M, McGill N (2018): The established and evolving role of nailfold capillaroscopy in connective tissue disease. Connective tissue disease- current state of art. Doi:10.5772/intechopen.82386.

11. Yen E, Singh R (2018): Lupus-an unrecognized leading cause of death in young females: a population-based study using nationwide death certificates, 2000-2015, Arthritis Rheum., 70 (8): 1251-1255.

12. Lu L, Hu C, Zhao Y, He L, Zhou J, Li H, Du Y et al. (2018): Shotgun Lipidomics Revealed Altered Profiles of Serum Lipids in Systemic Lupus Erythematosus Closely Associated with Disease Activity. Biomolecules, 8 (4): 105.

13. Maricq H (1981): Wide field capillary microscopy. Arthritis Rhum., 24 (9): 1159-65.

14. Tariq S, Tervaert J, Osman M (2019): Nailfold Capillaroscopy in Systemic Lupus Erythematosus (SLE): a Point-of-Care Tool That Parallels Disease Activity and Predicts Future Complications. Current Treatment Options in Rheumatology, 5: 336-345.

15. Parks C, Santos A, Barbhaiya M, Costenbader $K$ (2017): Understanding the role of environmental factors in the development of systemic lupus erythematosus. Best Pract Res Clin Rheumatol., 31 (3): 306-320.

16. Gustafsson J, Lindberg M, Gunnarsson I, Pettersson S, Elvin K, Öhrvik J et al. (2017): Excess atherosclerosis in systemic lupus erythematosus, -A matter of renal involvement: Case control study of 281 SLE patients and 281 individually matched population controls. PLoS ONE, 12(4): $\mathrm{e} 0174572$.

17. Khairy N, Ezzat Y, Naeem N, Taha R, Wesam $R$ (2017): Atherosclerosis biomarkers in female systemic lupus erythematosus patients with and without cardiovascular diseases. The Egyptian Rheumatologist, 39 (1): 7-12.

18. McMahon M, Skaggs B, Grossman J, Wong W, Sahakian L, Chen W et al. (2019): Comparison of PREDICTS atherosclerosis biomarker changes after initiation of new treatments in patients with SLE. Lupus Science \& Medicine, 6 (1): e000321.

19. Katz G, Smilowitz N, Blazer A, Clancy R, Buyon J P, Berger J (2019): Systemic Lupus Erythematosus and Increased Prevalence of Atherosclerotic Cardiovascular Disease in Hospitalized Patients, Mayo Clinic Proceedings, 94 (8): 1436-1443.

20. Doria A, Shoenfeld Y, Wu R, Gambari PF, Puato M, Ghirardello A et al. (2003): Risk factors for subclinical atherosclerosis in a prospective cohort of patients with systemic lupus erythematosus. Annals of the Rheumatic Diseases, 62(11): 1071-1077.

21. Soliman S, Labeeb A , Soltan G , Nofal D (2018): Intima media thickness measurement as a marker of subclinical atherosclerosis in systemic lupus erythematosus patients, Menoufia Medical Journal, 31 (3): 800-804.

22. Ragab O, Ashmawy A, Abdo M, Mokbel A (2011): Nail fold capillaroscopy in systemic lupus erythematosus. Egyptian Rheumatologist, 33 (1): 61-67.

23. Yuksel S, Yuksel E, Yenercag M, Soylu K, Zengin H , Gulel O et al. (2014): Abnormal nail fold capillaroscopic findings in patients with coronary slow flow phenomenon. Int J Clin Exp Med., 7 (4): 1052-1058.

24. Belibou C, Ancuţa C, Ancuța E, Filoș C, Chirieac R (2012): Carotid intima-media thickness and plaque as surrogate biomarkers of atherosclerosis among consecutive women with systemic lupus erythematosus. Rom J Morphol Embryol., 53 (1): 29-34.

25. Manzi S, Selzer F, Sutton-tyrrell $K$ et al. (1999): Prevalence and risk factors of carotid plaque in women with systemic lupus erythematosus. Arthritis Rheum., 42 (1): $51-60$. 